\title{
Long-term proton pump inhibitors and risk of gastric cancer development after treatment for Helicobacter pylori: a population-based study
}

\author{
Ka Shing Cheung, ${ }^{1}$ Esther W Chan, ${ }^{2}$ Angel Y S Wong ${ }^{2}$ Lijia Chen, ${ }^{1}$ Ian C K Wong, ${ }^{2,3}$ \\ Wai Keung Leung'
}

'Department of Medicine, The University of Hong Kong, Queen Mary Hospital, Hong Kong ${ }^{2}$ Department of Pharmacology and Pharmacy, Centre for Safe Medication Practice and Research, The University of Hong Kong, Hong Kong ${ }^{3}$ UCL School of Pharmacy, University College London London, UK

\section{Correspondence to}

DrWai Keung Leung, Department of Medicine, Queen Mary Hospital, 102 Pokfulam Road, Hong Kong; waikleung@ hku.hk

Received 1 June 2017 Revised 27 August 2017 Accepted 4 September 2017

Published Online First

31 October 2017

\section{Linked}

- http://dx.doi.org/10.1136/ gutjnl-2017-315629

CrossMark

To cite: Cheung KS, Chan EW, Wong AYS, et al. Gut 2018:67:28-35.

\section{ABSTRACT}

Objective Proton pump inhibitors (PPIs) is associated with worsening of gastric atrophy, particularly in Helicobacter pylori (HP)-infected subjects. We determined the association between PPIs use and gastric cancer (GC) among HP-infected subjects who had received HP therapy.

Designs This study was based on a territory-wide health database of Hong Kong. We identified adults who had received an outpatient prescription of clarithromycinbased triple therapy between year 2003 and 2012 .

Patients who failed this regimen, and those diagnosed to have GC within 12 months after HP therapy, or gastric ulcer after therapy were excluded. Prescriptions of PPIs or histamine-2 receptor antagonists (H2RA) started within 6 months before $\mathrm{GC}$ were excluded to avoid protopathic bias. We evaluated GC risk with PPIs by Cox proportional hazards model with propensity score adjustment. H2RA was used as a negative control exposure.

Result Among the 63397 eligible subjects, 153 $(0.24 \%)$ developed GC during a median follow-up of 7.6 years. PPIs use was associated with an increased $\mathrm{GC}$ risk (HR 2.44, 95\% Cl 1.42 to 4.20), while H2RA was not ( $H R=.72,95 \% \mathrm{Cl} 0.48$ to 1.07). The risk increased with duration of PPIs use (HR 5.04, 95\% Cl 1.23 to 20.61; $6.65,95 \% \mathrm{Cl} 1.62$ to 27.26 and $8.34,95 \% \mathrm{Cl} 2.02$ to 34.41 for $\geq 1$ year, $\geq 2$ years and $\geq 3$ years, respectively). The adjusted absolute risk difference for PPIs versus nonPPIs use was 4.29 excess $\mathrm{GC}(95 \% \mathrm{Cl} 1.25$ to 9.54$)$ per 10000 person-years.

Conclusion Long-term use of PPIs was still associated with an increased GC risk in subjects even after HP eradication therapy.

\section{INTRODUCTION}

Gastric cancer is the third leading cause of cancer-related mortality in the world. ${ }^{1}$ Although Helicobacter pylori eradication has been shown to reduce the risk of gastric cancer development by $33 \%-47 \%,{ }^{23}$ a considerable proportion of these individuals continues to progress to gastric cancer even after eradication of $H$. pylori. Apart from baseline gastric histology at the time of eradication, ${ }^{4}$ data are sparse on other modifiable risks of gastric cancer development, particularly on the role of concurrent medications.

Proton pump inhibitors (PPIs) have been among the most commonly prescribed medications in

\section{Significance of this study}

What is already known on this subject?

- Although Helicobacter pylori eradication has been shown to reduce the risk of gastric cancer development, a considerable proportion of these individuals continues to progress to gastric cancer even after successful eradication of $H$. pylori.

- Previous studies have shown that the risk of gastric cancer was increased by $43 \%$ among PPIs users but the major confounding factor, $H$. pylori, was not adjusted in these analyses and the causal relationship may be biased.

What are the new findings?

- Long-term PPIs use was associated with a 2.4-fold increase in gastric cancer risk in H. pylori-infected subjects who had received eradication therapy.

- The risk of gastric cancer increases with the dose and duration of PPIs use.

How might it impact on clinical practice in the foreseeable future?

- Physicians should exercise caution when prescribing long-term PPIs to $\mathrm{H}$. pylori-infected individuals even after successful eradication of H. pylori.

the world since the first PPI became available in the 1980s. ${ }^{5}$ Although PPIs are generally considered safe, recent data have demonstrated various adverse effects associated with long-term use of PPIs including bone fracture, ${ }^{6}$ Clostridium difficile infection, ${ }^{7}$ pneumonia, ${ }^{8}$ myocardial infarction and even stroke. ${ }^{9}$ Apart from the systemic adverse effects, there are also concerns on the long-term safety profile of PPIs in the stomach. The use of PPIs is associated with profound acid suppression, which could worsen atrophic gastritis. ${ }^{10}$ The risk is considerably high among individuals infected with H. pylori who are susceptible to the development of corpus atrophy. ${ }^{11}$ Moreover, PPIs stimulate the production of gastrin, which is a potent growth factor, and hypergastrinemia has been shown to induce hyperplasia of enterochromaffin-like cells. ${ }^{11}$ A recent meta-analysis showed that the 
risk of gastric cancer is increased by $43 \%$ among PPI users. ${ }^{12}$ However, these studies included both $H$. pylori-infected and $H$. pylori-negative subjects. Although previous short-term studies suggested the resolution of corpus atrophy with $H$. pylori eradication therapy in patients with GORD, ${ }^{13}{ }^{14}$ it remains uncertain whether the potential risk of PPIs on gastric cancer development could be eliminated by clearance of $H$. pylori.

This population-based study aimed to determine the risk of gastric cancer development among individuals who had received treatment for $H$. pylori with focus on the role of longterm PPIs.

\section{METHODS}

\section{Data source}

Data were retrieved from Clinical Data Analysis and Reporting System (CDARS) of the Hong Kong Hospital Authority. The Hospital Authority is the sole public healthcare provider for primary, secondary and tertiary health services through seven hospital clusters and covers $87 \%-94 \%$ of all secondary and tertiary care in Hong Kong with a population of around 7.3 million. ${ }^{15}$ Under this system, there are altogether 42 public hospitals, 47 specialist out-patient clinics and 73 general out-patient clinics. All essential clinical information including patients' demographics, hospitalisation, visits to outpatient clinics and emergency departments, diagnoses, laboratory results, procedures, prescriptions, dispensing of medications and death are recorded in CDARS, which is an electronic database managed by the hospital authority. This database was established in 1995 for both audit and research purposes. To protect patient's confidentiality, each patient is assigned a unique, anonymous patient identifier, which is linked to all the clinical data contained in CDARS. A number of high-quality, population-based studies ${ }^{16-18}$ and multinational pharmacovigilance studies ${ }^{19} 20$ have been conducted based on the data retrieved from CDARS. The International Classification of Diseases, Ninth Revision (ICD-9), was used for disease coding and previous studies have verified the accuracy of the coding in CDARS with high positive and negative predictive values of more than $90 \% .{ }^{1721}$ The study protocol was approved by the Institutional Review Board of the University of Hong Kong and the West Cluster of the Hong Kong Hospital Authority (reference no: UW 16-545).

\section{Study subjects}

We identified all adult patients who were aged 18 years or above and had been prescribed a minimum of 7-day course of clarithromycin-based triple therapy for $H$. pylori infection in outpatient clinics between 1 January 2003 and 31 December 2012. H. pylori infection was diagnosed by either upper endoscopy with biopsy-based tests or urea breath test in clinical practice, as serology and stool antigen tests were not available in local public hospitals. The prescription of clarithromycin-based triple therapy was identified by the co-prescription of one of the PPIs with clarithromycin and either amoxicillin or metronidazole, with doses as described previously. ${ }^{22}$ The start date of the prescriptions should be the same, with an overlapping duration of 7-14 days. Clarithromycin-based triple therapy was the first-line therapy for $H$. pylori in Hong Kong during the study period due to the low clarithromycin resistance rate $(8 \%)^{23}$ and overall high eradication rate $(>90 \%) .{ }^{24}$ To remove the confounding effects of symptoms from gastric cancer leading to the use of PPIs or histamine 2-receptor antagonist (H2RA) (ie, protopathic bias),
Patients receiving clarithromycin-based triple therapy between Jan 2003 and Dec 2012 ( $n=74,612)$

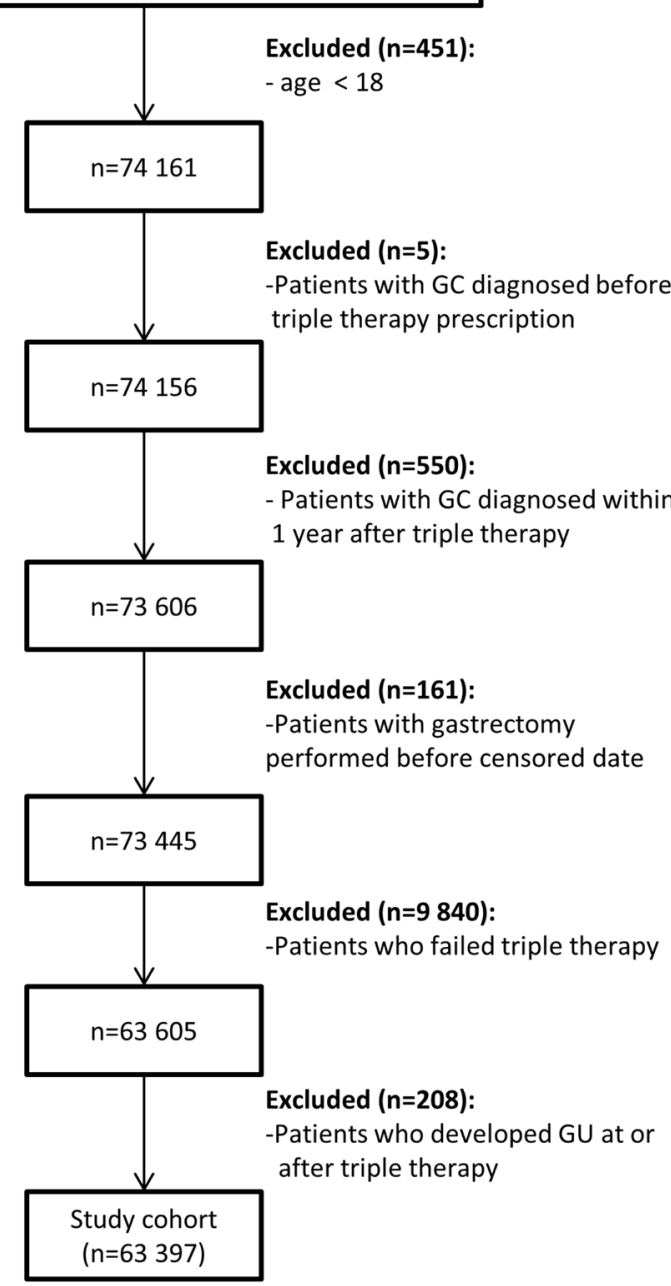

Figure 1 Study patient selection flow diagram. GC, gastric cancer; GU, gastric ulcer.

prescriptions of these agents started within 6 months prior to the gastric cancer diagnosis were excluded. ${ }^{25} 26$

Since gastric cancer can masquerade as non-healing ulcer, all patients with gastric ulcer diagnosed at the time of or any time after receiving triple therapy were excluded. As there may be a delay in the diagnosis of gastric cancer, patients who developed gastric cancer within the first year of $H$. pylori eradication therapy were also excluded. Patients with history of gastric cancer, previous gastrectomy or those who failed triple therapy were also excluded to ensure homogeneity of our study cohort. We defined failure of $H$. pylori eradication therapy as the requirement of subsequent prescriptions of (1) repeated course of clarithromycin-based triple therapy, (2) a second-line therapy (bismuth-based quadruple therapy or PPI-levofloxacin-amoxicillin) or (3) a third-line therapy (rifabutin-based therapy). Figure 1 illustrates the inclusion and exclusion process of patients in this study. The time frame of the study is shown in the efigure 1 in the online supplementary file 1 .

\section{Outcomes}

The primary outcome was the development of gastric adenocarcinoma. The observation period commenced from the date 
of first triple therapy prescription (ie, index date) and was censored at the date of diagnosis of gastric cancer, death or end of the study (31 December 2015). The date of diagnosis of gastric cancer was defined as the first date of hospitalisation for gastric cancer workup or treatment. Follow-up duration of individual patient was defined as the duration of observation between the index date and the censored date. All cases of gastric adenocarcinoma were identified in accordance with the ICD-9 (see etable 1 in the online supplementary file 1). We excluded patients with diagnosis of gastric lymphoma in this study. In order to ensure the validity of the case definition, a list of diagnostic codes was reviewed and finalised by a group of gastroenterologists.

\section{Study variables}

The primary exposure of interest was the subsequent prescription of PPIs after receiving the $H$. pylori eradication therapy. Potential confounders for gastric cancer development were also evaluated including the age of receiving triple therapy, sex, smoking status, alcohol consumption, history of gastric ulcer, history of duodenal ulcer, other comorbidities (including diabetes mellitus, hypertension, dyslipidaemia, obesity, ischaemic heart disease, atrial fibrillation, congestive heart failure, stroke, chronic renal failure and cirrhosis) and uses of various medications including statin, metformin, aspirin, non-steroidal anti-inflammatory drugs, cyclooxygenase-2 (COX-2) inhibitors, clopidogrel and H2RA.

PPIs are much more potent than H2RA in terms of gastric acid suppression, ${ }^{27}$ and previous studies did not reveal any association between gastric cancer development and H2RA. 252829 Hence, H2RA was selected as a negative control exposure in our study. If there is a positive association between H2RA and gastric cancer, this will suggest some unmeasured factors (including protopathic bias) that confound the causal relationship between PPIs and gastric cancer development.

To further control for possible confounding effects, another cohort of PPIs users (defined similarly as at least weekly use) who had not received $H$. pylori eradication therapy and fulfilled the same inclusion and exclusion criteria as in our $H$. pylori eradication cohort was recruited for comparison. These PPIs users who had not received $H$. pylori eradication therapy were then matched with the PPIs users who had received $H$. pylori eradication therapy $(\mathrm{n}=3271)$ by age $( \pm 5$ years $)$, sex, duration of follow-up ( \pm 2 years) and frequency of PPIs use $( \pm 0.3)$ in a 1:4 ratio. The incidence rates of gastric cancer in the two PPIs cohorts were compared.

We used similar approaches as adopted by Poulsen $e a^{28}$ to ascertain smoking status and alcohol consumption as these data were not available in the CDRAS. Smoking was identified by the ICD-9 code of V15.82 while chronic obstructive pulmonary disease (COPD) (ICD-9 codes: 491, 492, 496) was also used as proxy of heavy smoking. Heavy alcohol consumption was identified by alcohol-related diseases, including hepatic and gastrointestinal diseases, neurological and psychiatric diseases (ICD-9: $291,303,305.0,571,980)$. The diagnostic codes of other variables are listed in the etable 1 in the online supplementary file 1.

In the primary analysis, the exposure categories of various medications were categorised similarly into non-regular use (<weekly use; reference group) and regular use (at least weekly use) as described by Thrift et al..$^{30}$ The treatment duration of each prescription of a particular medication was defined as the difference between the prescription start date and end date within the observation period. The total treatment duration of that particular medication was then calculated by summing up the treatment duration of each prescription.

To study the dose-response relationship of PPIs on gastric cancer, the frequency of PPIs use was classified into three groups: (1) < weekly use, (2) weekly to <daily use and (3) daily use. The frequency of PPIs use was calculated by dividing the total treatment duration by the duration of follow-up. The effect of PPIs was also studied with regard to the duration of therapy which was categorised into $\geq 1$ year, $\geq 2$ years and $\geq 3$ years as defined in a recent meta-analysis. ${ }^{12}$

\section{Data validation}

As individual's identification is anonymised in the electronic database (CDARS), we could only retrieve detailed information of the gastric cancer cases that were managed in our centre (Queen Mary Hospital), which is a tertiary referral centre and a university teaching hospital. Of the 153 gastric cancer cases, 12 cases were managed in our centre and were reviewed in detail for gastric histology.

\section{Statistical analyses}

All statistical analyses were performed using R V.3.2.3 (R Foundation for Statistical Computing) statistical software. Continuous variables were expressed as median and IQR. Mann-Whitney U-test was used to compare continuous variables of two groups. $\chi^{2}$ test or Fisher's exact test was applied for categorical variables. The crude HR of gastric cancer development with PPIs use was calculated by univariate analysis using Cox proportional hazards model. For multivariable analysis, PPIs use and other covariates (age of receiving $H$. pylori eradication therapy, sex, smoking, alcohol use, comorbidities and concomitant medications) were included in the Cox model. To better control for the confounders, propensity score adjustment was performed. Propensity scores were derived from logistic regression to represent the conditional probability of PPIs use given the aforementioned covariates. To further reduce the bias from unmeasured confounding, individuals with extreme scores in the upper and lower tails of the propensity score distribution were excluded. ${ }^{31}$ In order to establish the cut-points for trimming, we constructed 20 categories of $5 \%$ each for the distribution of scores. To assess the balance of the continuous variable (age of receiving H. pylori eradication therapy) between PPIs users and non-users, the t-statistics adjusted for propensity score strata (by linear regression) and the $\mathrm{p}$ value were determined.

In the primary analysis, the first and 20th propensity score strata were trimmed, and the estimated propensity score was then used as an adjustment variable in the Cox proportional hazards model to derive the HR (propensity score adjustment with trimming). A sensitivity analysis was also performed without trimming the extreme propensity score strata (propensity score adjustment without trimming). In addition, the HR by univariate and multivariable analyses from Cox proportional hazards model were presented. For subgroup analysis, the risk of gastric cancer with PPIs use was stratified according to the tumour sites (cardia and non-cardia regions). Moreover, we estimated the propensity score adjusted absolute difference in gastric cancer risk for PPIs and non-PPIs use by the adjusted HR minus 1 , followed by the multiplication of the crude incidence rate among non-PPIs users. ${ }^{32}$ As H2RA was selected as a negative control exposure, propensity scores were also derived from logistic regression to represent the conditional probability of H2RA use given the other variables. The HR of gastric cancer with H2RA use was determined by propensity score adjustment 
Table 1 Characteristics of PPIs and non-PPIs users

\begin{tabular}{|c|c|c|c|}
\hline & All $(n=63397)$ & $\begin{array}{l}\text { PPIs } \\
\text { users }(n=3271)\end{array}$ & $\begin{array}{l}\text { Non-PPIs } \\
\text { users }(n=60126)\end{array}$ \\
\hline $\begin{array}{l}\text { Age at triple } \\
\text { therapy (years)* }\end{array}$ & $54.7(46.0-65.4)$ & $64.1(53.6-75.3)$ & $54.3(45.7-64.7)$ \\
\hline Male sex $(n, \%)$ & 29499 (46.5\%) & 1641 (50.2\%) & $27858(46.3 \%)$ \\
\hline $\begin{array}{l}\text { Duration of follow- } \\
\text { up (years)* }\end{array}$ & $7.6(5.1-10.3)$ & $7.4(4.5-10.0)$ & $7.6(5.2-10.3)$ \\
\hline Smoking $(\mathrm{n}, \%)$ & $1629(2.6 \%)$ & $162(5.0 \%)$ & $1467(2.4 \%)$ \\
\hline Alcohol (n, \%) & $552(0.9 \%)$ & $50(1.5 \%)$ & $502(0.8 \%)$ \\
\hline Dyspepsia (n, \%) & 4145 (6.5\%) & $262(8.0 \%)$ & $3883(6.5 \%)$ \\
\hline GORD $(n, \%)$ & $3278(5.2 \%)$ & $593(18.1 \%)$ & 2685 (4.5\%) \\
\hline History of GU $(n, \%)$ & $1268(2.0 \%)$ & $153(4.7 \%)$ & $1115(1.9 \%)$ \\
\hline History of DU $(n, \%)$ & $1897(3.0 \%)$ & $139(4.2 \%)$ & $1758(2.9 \%)$ \\
\hline $\mathrm{DM}(\mathrm{n}, \%)$ & $7383(11.6 \%)$ & $772(23.6 \%)$ & $6611(11.0 \%)$ \\
\hline Hypertension ( $\mathrm{n}, \%$ ) & 13065 (20.6\%) & $1334(40.8 \%)$ & 11731 (19.5\%) \\
\hline $\begin{array}{l}\text { Dyslipidaemia } \\
(\mathrm{n}, \%)\end{array}$ & $5045(8.0 \%)$ & 579 (17.7\%) & 4466 (7.4\%) \\
\hline Obesity & $637(1.0 \%)$ & $61(1.9 \%)$ & $576(1.0 \%)$ \\
\hline $\operatorname{IHD}(\mathrm{n}, \%)$ & $5701(9.0 \%)$ & $906(27.7 \%)$ & 4795 (8.0\%) \\
\hline $\mathrm{AF}(\mathrm{n}, \%)$ & $2404(3.8 \%)$ & $371(11.3 \%)$ & $2033(3.4 \%)$ \\
\hline CHF $(n, \%)$ & $2512(4.0 \%)$ & $463(14.2 \%)$ & 2049 (3.4\%) \\
\hline Stroke $(n, \%)$ & 3965 (6.3\%) & $561(17.2 \%)$ & $3404(5.7 \%)$ \\
\hline $\operatorname{CRF}(\mathrm{n}, \%)$ & $1388(2.2 \%)$ & $236(7.2 \%)$ & $1152(1.9 \%)$ \\
\hline Cirrhosis (n, \%) & 1037 (1.6\%) & $98(3.0 \%)$ & $939(1.6 \%)$ \\
\hline Statins (n, \%) & $13180(20.8 \%)$ & $1351(41.3 \%)$ & 11829 (19.7\%) \\
\hline Metformin (n, \%) & 7935 (12.5\%) & $605(18.5 \%)$ & 7330 (12.2\%) \\
\hline Aspirin (n, \%) & 8965 (14.1\%) & $1358(41.5 \%)$ & $7607(12.7 \%)$ \\
\hline $\begin{array}{l}\text { NSAIDs/COX-2 } \\
\text { inhibitors }(n, \%)\end{array}$ & $3556(5.6 \%)$ & $391(12.0 \%)$ & $3165(5.3 \%)$ \\
\hline Clopidogrel (n, \%) & 980 (1.5\%) & $200(6.1 \%)$ & $780(1.3 \%)$ \\
\hline H2RA $(n, \%)$ & $21729(34.3 \%)$ & $1499(45.8 \%)$ & $20230(33.6 \%)$ \\
\hline
\end{tabular}

Categorical variables were expressed as number (\%).

Drug use was defined as at least weekly use, and expressed as number (\%).

${ }^{*}$ Age was expressed as median (years) with IQR.

$A F$, atrial fibrillation; $C H F$, congestive heart failure; COX-2, cyclooxygenase-2; $C R F$, chronic renal failure; DM, diabetes mellitus; DU, duodenal ulcer; GU, gastric ulcer; H2RA, histamine 2 receptor antagonist; IHD, ischaemic heart disease; NSAIDs, nonsteroidal anti-inflammatory drugs; PPIs, proton pump inhibitors.

after trimming. All statistical tests were two-sided, and a $\mathrm{p}$ value of $<0.05$ was used to define statistical significance.

\section{RESULTS}

\section{Patient characteristics}

A total of 74612 subjects received clarithromycin-based triple therapy during the 10 -year period. After excluding patients who did not fulfil our inclusion criteria (figure 1), 63397 subjects were included in the final analysis. The median age of this cohort at the time of $H$. pylori eradication therapy was 54.7 years (IQR 46.0-65.4 years) and $46.5 \%$ were men. The median follow-up was 7.6 years (IQR 5.1-10.3 yeas) and the total follow-up duration was 483260 person-years. The baseline characteristics of the whole cohort and the subgroups according to PPIs and H2RA use are shown in tables 1 and 2. Notably, both PPIs users (64.1 vs 54.3 years) and H2RA users (60.0 vs 52.0 years) were older than the corresponding non-users.

\section{Risk of gastric cancer development}

One hundred and fifty-three (0.24\%) subjects developed gastric cancer after $H$. pylori eradication therapy. Among them, $31(20.3 \%)$ cancer were in the cardia and $95(62.1 \%)$ in the
Table 2 Characteristics of H2RA and non-H2RA users

\begin{tabular}{|c|c|c|c|}
\hline & All $(n=63397)$ & $\begin{array}{l}\text { H2RA } \\
\text { users }(n=21729)\end{array}$ & $\begin{array}{l}\text { Non-H2RA } \\
\text { users }(n=41668)\end{array}$ \\
\hline $\begin{array}{l}\text { Age at triple } \\
\text { therapy (years)* }^{*}\end{array}$ & $54.7(46.0-65.4)$ & $60.0(51.6-71.0)$ & $52.0(43.4-61.6)$ \\
\hline Male sex (n, \%) & 29499 (46.5\%) & 9454 (43.5\%) & 20045 (48.1\%) \\
\hline $\begin{array}{l}\text { Duration of follow- } \\
\text { up (years)* }\end{array}$ & $7.6(5.1-10.3)$ & $7.2(4.8-9.8)$ & $7.8(5.3-10.5)$ \\
\hline Smoking (n, \%) & $1629(2.6 \%)$ & $863(4.0 \%)$ & 766 (1.8\%) \\
\hline Alcohol (n, \%) & $552(0.9 \%)$ & $232(1.1 \%)$ & $320(0.8 \%)$ \\
\hline Dyspepsia (n, \%) & $4145(6.5 \%)$ & $1826(8.4 \%)$ & $2319(5.6 \%)$ \\
\hline GORD $(n, \%)$ & $3278(5.2 \%)$ & $1629(7.5 \%)$ & $1649(4.0 \%)$ \\
\hline $\begin{array}{l}\text { History of GU } \\
(n, \%)\end{array}$ & $1268(2.0 \%)$ & $446(2.1 \%)$ & $822(2.0 \%)$ \\
\hline $\begin{array}{l}\text { History of DU } \\
(n, \%)\end{array}$ & $1897(3.0 \%)$ & $503(2.3 \%)$ & $1394(3.3 \%)$ \\
\hline $\mathrm{DM}(\mathrm{n}, \%)$ & $7383(11.6 \%)$ & 3885 (17.9\%) & $3498(8.4 \%)$ \\
\hline $\begin{array}{l}\text { Hypertension } \\
(n, \%)\end{array}$ & $13065(20.6 \%)$ & $7137(32.8 \%)$ & $5928(14.2 \%)$ \\
\hline $\begin{array}{l}\text { Dyslipidaemia } \\
(\mathrm{n}, \%)\end{array}$ & 5045 (8.0\%) & 2939 (13.5\%) & 2106 (5.1\%) \\
\hline Obesity & $637(1.0 \%)$ & $351(1.6 \%)$ & $286(0.7 \%)$ \\
\hline $\operatorname{IHD}(n, \%)$ & $5701(9.0 \%)$ & $3560(16.4 \%)$ & $2141(5.1 \%)$ \\
\hline $\operatorname{AF}(n, \%)$ & $2404(3.8 \%)$ & $1468(6.8 \%)$ & $936(2.2 \%)$ \\
\hline CHF $(n, \%)$ & $2512(4.0 \%)$ & $1512(7.0 \%)$ & $1000(2.4 \%)$ \\
\hline Stroke $(n, \%)$ & $3965(6.3 \%)$ & $2466(11.3 \%)$ & $1499(3.6 \%)$ \\
\hline $\operatorname{CRF}(\mathrm{n}, \%)$ & $1388(2.2 \%)$ & $814(3.7 \%)$ & $574(1.4 \%)$ \\
\hline Cirrhosis (n, \%) & $1037(1.6 \%)$ & $425(2.0 \%)$ & $612(1.5 \%)$ \\
\hline Statins (n, \%) & $13180(20.8 \%)$ & 7401 (34.1\%) & $5779(13.9 \%)$ \\
\hline Metformin (n, \%) & 7935 (12.5\%) & 3899 (17.9\%) & $4036(9.7 \%)$ \\
\hline Aspirin (n, \%) & 8965 (14.1\%) & $6376(29.3 \%)$ & $2589(6.2 \%)$ \\
\hline $\begin{array}{l}\text { NSAIDs/COX-2 } \\
\text { inhibitors (n, \%) }\end{array}$ & 3556 (5.6\%) & 3092 (14.2\%) & 464 (1.1\%) \\
\hline Clopidogrel (n, \%) & $980(1.5 \%)$ & $602(2.8 \%)$ & $378(0.9 \%)$ \\
\hline PPIs (n, \%) & 3271 (5.2\%) & 1499 (6.9\%) & $1772(4.3 \%)$ \\
\hline
\end{tabular}

Categorical variables were expressed as number (\%).

Drug use was defined as at least weekly use and expressed as number (\%).

*Age was expressed as median (years) with IQR.

$A F$, atrial fibrillation; CHF, congestive heart failure; COX-2, cyclooxygenase-2; CRF, chronic renal failure; DM, diabetes mellitus; $\mathrm{DU}$, duodenal ulcer; GU, gastric ulcer; H2RA, histamine 2 receptor antagonist; IHD, ischaemic heart disease; NSAIDS, nonsteroidal anti-inflammatory drugs; PPIs, proton pump inhibitors.

non-cardia regions. The sites were not specified in the remaining 27 (17.6\%) cases (ICD-9: 151.9). Similar ratio were observed for all the stomach cancer cases $(n=12898)$ diagnosed in the public hospitals in Hong Kong during the study period (13.4\% in cardia, $67.5 \%$ in non-cardia and $19.1 \%$ cases with sites unspecified).

The histology reports of 12 (7.8\% out of 153$)$ gastric cancer cases from our centre were retrieved, and all cancers were verified to be adenocarcinoma. All patients were negative for $\mathrm{H}$. pylori on gastric biopsies at the time of diagnosis and had underlying chronic gastritis, while intestinal metaplasia was reported in five cases. For the degree of differentiation, five cases were poorly differentiated, three were moderately to poorly differentiated, one was moderately differentiated, while the degree of differentiation was unspecified for the remaining three. According to the Lauren classification, three cases were diffuse type, three were intestinal type and the histological subtypes were unspecified for the remaining six.

The median age at cancer diagnosis was 71.4 years (IQR 61.181.5 years). Patients who developed gastric cancer received $H$. 
Table 3 Association between PPIs use and risk of gastric cancer for the whole cohort and according to gastric cancer sites (non-cardia and cardia regions)

\begin{tabular}{|c|c|c|c|c|c|c|c|c|c|c|c|c|}
\hline \multirow{2}{*}{$\begin{array}{l}\text { PPIs frequency } \\
\text { All GC }\end{array}$} & \multicolumn{3}{|c|}{$\begin{array}{l}\text { Univariate } \\
\text { analysis }(n=63397, \mathrm{GC}=153)\end{array}$} & \multicolumn{3}{|c|}{$\begin{array}{l}\text { Multivariable } \\
\text { analysis }(n=63397, \mathrm{GC}=153)\end{array}$} & \multicolumn{3}{|c|}{$\begin{array}{l}\text { PS adjustment without } \\
\text { trimming }(n=63397, G C=153)\end{array}$} & \multicolumn{3}{|c|}{$\begin{array}{l}\text { PS adjustment with } \\
\text { trimming }(n=57057, G C=139)\end{array}$} \\
\hline & HR & $95 \% \mathrm{Cl}$ & p Value & HR & $95 \% \mathrm{Cl}$ & p Value & HR & $95 \% \mathrm{Cl}$ & p Value & HR & $95 \% \mathrm{Cl}$ & p Value \\
\hline $\begin{array}{l}\text { Non-user } \\
\text { (<weekly use) }\end{array}$ & Ref & - & - & Ref & - & - & Ref & - & - & Ref & - & - \\
\hline At least weekly & 2.80 & 1.73 to 4.52 & 0.003 & 2.19 & 1.31 to 3.66 & 0.003 & 2.14 & 1.27 to 3.58 & 0.004 & 2.44 & 1.42 to 4.20 & 0.002 \\
\hline \multirow[t]{2}{*}{ Non-cardia GC } & \multicolumn{3}{|c|}{$(n=63366, G C=122)$} & \multicolumn{3}{|c|}{$(n=63366, G C=122)$} & \multicolumn{3}{|c|}{$(n=63366, G C=122)$} & \multicolumn{3}{|c|}{$(n=57028, G C=112)$} \\
\hline & $H R$ & $95 \% \mathrm{Cl}$ & p Value & $H R$ & $95 \% \mathrm{Cl}$ & p Value & $H R$ & $95 \% \mathrm{Cl}$ & p Value & $H R$ & $95 \% \mathrm{Cl}$ & $p$ Value \\
\hline $\begin{array}{l}\text { Non-user } \\
\text { (<weekly use) }\end{array}$ & Ref & - & - & Ref & - & - & Ref & - & - & Ref & - & - \\
\hline At least weekly & 2.98 & 1.76 to 5.05 & 0.001 & 2.56 & 1.46 to 4.49 & 0.001 & 2.43 & 1.38 to 4.28 & 0.002 & 2.59 & 1.42 to 4.72 & 0.002 \\
\hline \multirow[t]{2}{*}{ Cardia GC } & \multicolumn{3}{|c|}{$(n=63275, G C=31)$} & \multicolumn{3}{|c|}{$(n=63275, G C=31)$} & \multicolumn{3}{|c|}{$(n=63275, G C=31)$} & \multicolumn{3}{|c|}{$(n=56947, G C=27)$} \\
\hline & $\mathrm{HR}$ & $95 \% \mathrm{Cl}$ & p Value & $\mathrm{HR}$ & $95 \% \mathrm{Cl}$ & p Value & $\mathrm{HR}$ & $95 \% \mathrm{Cl}$ & p Value & $\mathrm{HR}$ & $95 \% \mathrm{Cl}$ & p Value \\
\hline $\begin{array}{l}\text { Non-user } \\
\text { (<weekly use) }\end{array}$ & Ref & - & - & Ref & - & - & Ref & - & - & Ref & - & - \\
\hline At least weekly & 2.10 & 0.64 to 6.90 & 0.222 & 1.24 & 0.35 to 4.34 & 0.736 & 1.26 & 0.35 to 4.52 & 0.722 & 1.97 & 0.57 to 6.82 & 0.286 \\
\hline
\end{tabular}

Significant $\mathrm{p}$ values were highlighted in bold.

GC, gastric cancer; PPIs, proton pump inhibitors; PS, propensity score.

pylori eradication therapy at a median age of 65.4 years (IQR 56.4-76.2 years), and the median time from $H$. pylori eradication therapy to cancer development was 4.9 years (IQR 2.7-7.2 years). The overall incidence rate of gastric cancer in this cohort was 3.2 per 10000 person-years. The incidence rate of gastric cancer of PPIs users for each follow-up year is shown in etable 2 in the online supplementary file 1 , which ranged from 0 to 18.5 per 10000 person-years. Patients who developed gastric cancer within the first year of $H$. pylori eradication therapy were excluded in this study as described above.

\section{Association of PPIs use and risk of gastric cancer}

Table 3 shows the associations between PPIs use and gastric cancer development after $H$. pylori therapy. PPIs users (at least weekly use) were found to have a higher risk of gastric cancer (HR 2.44, 95\% CI 1.42 to 4.20 ) after propensity score adjustment with trimming. Sensitivity analysis confirms the association of PPIs use with gastric cancer development by either multivariable analysis (HR 2.19, 95\% CI 1.31 to 3.66 ) or propensity score adjustment without trimming (HR 2.14, 95\% CI 1.27 to 3.58 ).

The propensity score adjusted absolute risk difference between PPIs use and non-PPIs use was 4.29 excess gastric cancer $(95 \% \mathrm{CI}$ 1.25 to 9.54$)$ per 10000 person-years.
After stratification by the site of tumour, PPIs use was only found to be significantly associated with an increased risk of non-cardia gastric cancer (HR 2.59, 95\% CI 1.42 to 4.72 ) but not cardia cancer (HR 1.97, 95\% CI 0.57 to 6.82 ). Sensitivity analysis yielded similar results.

PPIs users were older than non-PPIs users by around 10 years, and the HR of gastric cancer with increasing age was 1.06 on multivariable analysis. Therefore, the t-statistics was used to assess the balance of age between the two groups. After adjusting for propensity score strata, the age was balanced between the two groups ( $\mathrm{t}$-statistics $-1.42, \mathrm{p}=0.156)$.

\section{Frequency and duration of PPIs use on risk of gastric cancer}

A total of $3271(5.2 \%)$ patients in this cohort had used PPIs and the median duration of PPIs use was 2.7 years (IQR 1.5-5.1 years). Among them, 19 (0.6\%) developed gastric cancer $(8.1$ per 10000 person-years). We further determined the frequency and duration of PPIs use on gastric cancer development. Patients were first stratified according to the frequency of PPIs use (table 4) into three groups as described in the Methods section. When compared with the reference group (<weekly use), there was a progressive increase in the risk of gastric cancer with more frequent use of PPIs (HR 2.43, 95\% CI 1.37 to 4.31 for 'weekly

Table 4 HRs and 95\% Cls for the association between frequency and duration of PPIs use and risk of gastric cancer (propensity score adjustment with trimming)

\begin{tabular}{|c|c|c|c|c|c|c|c|c|c|}
\hline \multirow[b]{2}{*}{ PPIs frequency } & \multicolumn{9}{|c|}{ Dose-response relationship $(\mathrm{n}=57057, \mathrm{GC}=139)$} \\
\hline & \multicolumn{3}{|c|}{ HR } & \multicolumn{3}{|c|}{$95 \% \mathrm{Cl}$} & \multicolumn{3}{|c|}{$\mathrm{p}$ Value } \\
\hline Non-user (<weekly use) & \multicolumn{3}{|l|}{ Ref } & \multicolumn{3}{|l|}{-} & \multicolumn{3}{|l|}{-} \\
\hline Daily & \multicolumn{3}{|l|}{4.55} & \multicolumn{3}{|c|}{1.12 to 18.52} & \multicolumn{3}{|l|}{0.034} \\
\hline \multirow[t]{2}{*}{ PPIs frequency } & \multicolumn{3}{|c|}{ PPIs use $\geq 1$ year $(n=50932, \mathrm{GC}=112)$} & \multicolumn{3}{|c|}{ PPIs use $\geq 2$ years $(n=49462, G C=88)$} & \multicolumn{3}{|c|}{ PPIs use $\geq 3$ years $(\mathrm{n}=48511, \mathrm{GC}=69)$} \\
\hline & $H R$ & $95 \% \mathrm{Cl}$ & $\mathrm{p}$ Value & HR & $95 \% \mathrm{Cl}$ & $\mathrm{p}$ Value & $\mathrm{HR}$ & $95 \% \mathrm{Cl}$ & $\mathrm{p}$ Value \\
\hline Daily & 5.04 & $1.23-20.61$ & 0.024 & 6.65 & $1.62-27.26$ & 0.009 & 8.34 & $2.02-34.41$ & 0.004 \\
\hline
\end{tabular}

Significant $\mathrm{p}$ Values were highlighted in bold.

GC, gastric cancer; PPIs, proton pump inhibitors. 
to < daily use' and HR $4.55,95 \%$ CI 1.12 to 18.52 for 'daily use'). Sensitivity analysis yielded similar results (see etables 3 and 4 in the online supplementary file 1).

Furthermore, the effect of long-term PPIs on gastric cancer development was studied with regard to the duration of PPIs therapy ( $\geq 1$ year, $\geq 2$ years and $\geq 3$ years). As shown in table 4 , the risk increased with longer duration of PPIs use (HR 5.04, $95 \%$ CI 1.23 to 20.61 for $\geq 1$ year of use; HR $6.65,95 \%$ CI 1.62 to 27.26 for $\geq 2$ years of use and HR $8.34,95 \%$ CI 2.02 to 34.41 for $\geq 3$ years of use).

\section{Association of H2RA use and risk of gastric cancer}

To test for potential confounding, H2RA was used as a negative control exposure. The HR of gastric cancer with H2RA use on univariate analysis was 0.95 (95\% CI 0.67 to 1.33 ), while the HR from propensity score adjustment with trimming was 0.72 (95\% CI 0.48 to 1.07 ).

\section{Comparison of the incidence rates of gastric cancer with a matched cohort of PPIs users who had not received $H$. pylori eradication therapy}

To further check for potential confounding, another cohort of PPIs users (at least weekly use) who had not received $H$. pylori eradication therapy were included for comparison. Altogether, 142460 PPIs users without prior $H$. pylori eradication therapy were identified with a total of 705094 person-years of follow-up. Among them, there were 59 gastric cancer cases making a crude incidence rate of 0.8 cases per 10000 person-years. After matching, the incidence rate was 8.1 and 1.0 cases per 10000 person-years in the two cohorts of PPIs users with and without H. pylori eradication therapy, respectively (incidence rate ratio 0.12 ; $95 \%$ CI 0.05 to 0.26 ) (table 5).

\section{DISCUSSION}

In this population-based study that addressed the risk of gastric cancer development in $H$. pylori-infected individuals after receiving eradication treatment, we found that long-term use of PPIs increased the risk of gastric cancer development. Our results showed that even after apparent successful $H$. pylori eradication therapy, those who used long-term PPIs had a 2.4-fold increase in the risk of gastric cancer development than non-users. This increase in risk was not observed among H2RA users. Further analysis demonstrated a dose-dependent and time-dependent increase in the HRs of gastric cancer with PPIs use, with the highest risk observed in daily users of PPIs (HR 4.55). Patients who took PPIs daily for $\geq 3$ years were at the highest risk (HR 8.34). Notably, the increase in HR was limited to non-cardia cancer, although this result should be interpreted with caution as this subgroup analysis has a relatively small number of cardia cancers.

Gastric atrophy is considered to be a precursor of gastric cancer, which is usually associated with chronic $H$. pylori infection. While PPIs are potent acid suppressors, there have been concerns on the possible worsening of gastric atrophy by longterm PPIs and the associated increase in gastric cancer risk. ${ }^{10}{ }^{12}$ Most published data supported that long-term PPIs could worsen corpus gastritis and atrophy, particularly in $H$. pylori-positive subjects. ${ }^{1033}$ Although the long-term use of PPIs for more than 12 months was shown to be associated with an increased risk of gastric cancer ${ }^{12}$ these results are largely confounded by the unknown prevalence of $H$. pylori in the study population. ${ }^{25} 2829$ On the other hand, treatment of $H$. pylori in patients with reflux oesophagitis requiring long-term PPIs was found to eliminate gastric mucosal inflammation and possibly induce regression of corpus glandular atrophy. ${ }^{13}$ Hence, current guideline recommends eradication of $H$. pylori prior to the initiation of long-term PPIs. ${ }^{34}$ While GORD is related to overproduction of gastric acid and hence a lower prevalence of corpus atrophy, these patients may not be the ideal population to study relationship between PPIs use and worsening of corpus atrophy and gastric cancer. There is so far no long-term data to support that $H$. pylori eradication is sufficient in preventing cancer development in these individuals who use long-term PPIs.

To our knowledge, this is the first study to demonstrate that long-term PPIs use, even after $H$. pylori eradication therapy, is still associated with an increased risk of gastric cancer. This is likely related to the profound acid suppression of PPIs that worsens atrophic gastritis, particularly in those patients with established gastric atrophy as a result of chronic $H$. pylori-induced inflammation. The lack of association between H2RA use and gastric cancer development further supports the specific role of PPIs on gastric cancer development. One of the strengths of our study is the use of data from large population-based database with complete information on subsequent diagnoses and drug prescriptions, thus minimising the selection, information and recall biases. As all medications are dispensed by the

Table 5 Comparison of incidence rates of gastric cancer in different cohorts according to PPIs uses and prior Helicobacter pylori (HP) eradication therapy

\begin{tabular}{llllll}
\hline Before matching & Number of patients & Number of person-years & Number of $\mathrm{GC}$ cases & $\begin{array}{l}\text { Incidence rate (per } \\
10000 \text { person-years) }\end{array}$ & $\begin{array}{l}\text { Incidence rate ratio with } \\
95 \% \mathrm{Cl}\end{array}$ \\
\hline $\begin{array}{l}\text { Non-PPIs users with prior HP } \\
\text { therapy }\end{array}$ & 60126 & 459864 & 134 & 2.9 & Ref \\
$\begin{array}{l}\text { PPIs users with prior HP } \\
\text { therapy }\end{array}$ & 3271 & 23395 & 19 & 8.1 & $2.81(1.68$ to 4.43$)$ \\
$\begin{array}{l}\text { PPIs users without prior HP } \\
\text { therapy }\end{array}$ & 142460 & 705094 & 59 & 0.8 & $0.29(0.21$ to 0.39$)$ \\
$\begin{array}{l}\text { After matching } \\
\text { Number of patients }\end{array}$ & Number of person-years & Number of GC cases & $\begin{array}{l}\text { Incidence rate (per } 10000 \\
\text { person-years) }\end{array}$ & $\begin{array}{l}\text { Incidence rate ratio with } \\
95 \% \mathrm{Cl}\end{array}$ \\
$\begin{array}{l}\text { PPIs users with prior HP } \\
\text { therapy }\end{array}$ & 3270 & 23384 & 19 & 8.1 & Ref \\
$\begin{array}{l}\text { PPIs users without prior HP } \\
\text { therapy* }\end{array}$ & 13080 & 93500 & 9 & 1.0 & $0.12(0.05$ to 0.26$)$
\end{tabular}

* Matched with age ( \pm 5years), sex, duration of follow-up ( \pm 2 years) and frequency of PPIs use $( \pm 0.3)$ in a 1:4 ratio.

$\mathrm{GC}$, gastric cancer; PPIs, proton pump inhibitors. 
hospital pharmacy at a very low cost to patients (ie, $£ 1$ per item for 16 weeks), the prescription records are generally identical to dispensing records. The large sample size and the relatively long duration of follow-up (median 7.6 years) allow for more precise effect estimation of gastric cancer risk attributed to various factors and enable subgroup analysis. The association was also consistent in both the frequency and duration of PPIs treatment, demonstrating a dose-response and time-response trend to suggest a cause-effect relationship.

Another strength of this study was the use of a strict exclusion criteria as well as propensity score adjustment to control for potential confounders in determining the causal relationship between PPIs use and gastric cancer development. The results remained significant by various sensitivity analyses. In addition, we recruited patients with successful $H$. pylori eradication only. In fact, failure to adjust for $H$. pylori infection is one of the major concerns in studying the effect of PPIs on gastric cancer risk in previous studies. ${ }^{25} 2829$ The indication bias and protopathic bias was another major concern that leads to the undetermined conclusion of the causal relationship between PPIs use and gastric cancer development in previous studies. ${ }^{25} 2829$ First, as gastric cancer can present with dyspepsia leading to an increase use of PPIs, all prescriptions of PPIs in the 6 months preceding the diagnosis of gastric cancer were excluded to avoid protopathic bias in this study. We used 6 months as the priori cut-off because previous study that specifically addressed the issue of protopathic bias showed that this was the most appropriate lag-time to be applied for the assessment of PPIs exposure on gastric cancer risk in pharmaco-epidemiological studies. ${ }^{26}$ Moreover, PPIs are not approved as first-line therapy for dyspepsia in the Hong Kong Hospital Authority, and H2RAs are usually the recommended treatment for this indication. One would anticipate a similar increase in gastric cancer risk among those taking H2RAs (negative control exposure) if there was significant indication bias in this cohort. The minimisation of protopathic bias and indication bias was further supported by the findings that the matched cohort of PPIs users without $H$. pylori eradication therapy had the lowest incidence rate when compared with the two post- $H$. pylori eradicated cohorts (table 5). By comparing the incidence rate of gastric cancer of a matched cohort of PPIs users who had not received $H$. pylori eradication therapy, we showed that $H$. pylori infection, even prior infection, was a more important factor than PPIs use in determining gastric cancer risk. PPIs increase the risk of gastric cancer development likely in the context of underlying $H$. pylori-associated chronic gastritis and atrophy. In addition, we excluded patients who had active gastric ulcer diagnosed at the time of $H$. pylori eradication therapy or during surveillance intervals as gastric cancer may masquerade as non-healing gastric ulcer.

Our study has several limitations. First, the information of some risk factors (eg, diet, family history and socioeconomic status) could not be obtained from the electronic database. Moreover, the identification of certain parameters (smoking, alcohol use and obesity) via coding may underestimate their true prevalence, as only patients who had heavy consumption of smoking and alcohol or who were morbidly obese would be coded. Second, although patients who failed triple therapy were identified by the repeated prescription of clarithromycin-based triple therapy or prescription of second-line and third-line therapies, it remains possible that a small proportion of patients who failed $H$. pylori eradication therapy might be missed. In this study, about $13 \%$ of patients received a second course of eradication therapy, which is compatible with the observed success rate of clarithromycin-based triple therapy in our population with relatively low prevalence of clarithromycin resistance during the study period. ${ }^{23}$ In addition, we have validated the negative H. pylori status of all 12 gastric cancer cases from our hospital. Third, although we included more than $63000 \mathrm{H}$. pylori-infected subjects, the small number of gastric cancer cases did not allow for any meaningful evaluation of the dosage effect and role of different PPIs. However, it was recently shown that there was no difference in the gastric cancer risk between longer and shorter-acting PPIs. ${ }^{35}$ Fourth, PPIs users may have a higher chance to have endoscopy than non-PPIs users resulting in discovery of more gastric cancers due to surveillance bias. However, as shown in the etable 2 in the online supplementary file 1 , the incidence rate of gastric cancer remained relatively stable throughout the follow-up period rather than an early peak in the first few years followed by a rapid drop in the ensuing years. Fifth, PPIs users were older than non-users in our cohort. Age was shown to be a significant risk factor for gastric cancer development (HR 1.06) on multivariable analysis. The comparability of the two groups may therefore be a concern, but this issue has been addressed by the use of propensity score adjustment with trimming and various sensitivity analyses. The non-significance of t-statistics between the PPIs users and non-users after adjusting for the propensity score strata further supports the robustness of this adjustment. Moreover, H2RA users were also significantly older than non-users (table 2), but a similar increase in gastric cancer risk was not observed among H2RA users. Sixth, our patients are mainly Chinese, and hence our results may not be generisable to other ethnic groups, as Asians are at a higher gastric cancer risk than the western population. ${ }^{1}$ Lastly, the detailed histological findings of gastric biopsies at baseline and at the time of gastric cancer development were not available in the CDARS, precluding more in-depth analysis between the association of PPIs and baseline histology on gastric cancer development.

\section{CONCLUSION}

Long-term use of PPIs in subjects with prior $\mathrm{H}$. pylori eradication was still associated with an increased risk of gastric cancer development, particularly for non-cardia cancer. There was also a clear dose-response and time-response trend of PPIs uses and gastric cancer risk. Physicians should therefore exercise caution when prescribing long-term PPIs to these patients even after successful eradication of $H$. pylori.

Contributors KSC, ICKW and WKL were involved with the study concept and design, analysis and interpretation of data, drafting of manuscript and approval of the final version of the manuscript. EWC, AYSW and LC were involved with acquisition of data, critical revision of the manuscript for important intellectual content and approval of the final version of the manuscript. ICKW and WKL were involved in the critical revision of the manuscript for important intellectual content and study supervision.

Competing interests WKL has received honorarium for attending advisory board meetings of Takeda and Abbott Laboratories.

Ethics approval Institutional Review Board of the University of Hong Kong and the West Cluster of Hospital Authority, Hong Kong.

Provenance and peer review Not commissioned; externally peer reviewed.

(c) Article author(s) (or their employer(s) unless otherwise stated in the text of the article) 2018. All rights reserved. No commercial use is permitted unless otherwise expressly granted.

\section{REFERENCES}

1 World Health Organisation. Cancer fact sheets: stomach cancer. http://gco.iarc.fr/ today/fact-sheets-cancers? cancer $=5 \&$ type $=0 \&$ sex $=0$.

2 Lee YC, Chiang TH, Chou CK, et al. Association between Helicobacter pylori eradication and gastric cancer incidence: a systematic review and meta-analysis. Gastroenterology 2016;150:1113-24. 
3 Ford AC, Forman D, Hunt RH, et al. Helicobacter pylori eradication therapy to prevent gastric cancer in healthy asymptomatic infected individuals: systematic review and meta-analysis of randomised controlled trials. BMJ 2014;348:g3174.

4 Wong BC, Lam SK, Wong WM, et al. Helicobacter pylori eradication to prevent gastric cancer in a high-risk region of China: a randomized controlled trial. JAMA 2004;291:187-94.

5 Forgacs I, Loganayagam A. Overprescribing proton pump inhibitors. BMJ 2008;336:2-3.

6 Yang $Y X$, Lewis JD, Epstein S, et al. Long-term proton pump inhibitor therapy and risk of hip fracture. JAMA 2006;296:2947-53.

7 Janarthanan S, Ditah I, Adler DG, et al. Clostridium difficile-associated diarrhea and proton pump inhibitor therapy: a meta-analysis. Am J Gastroenterol 2012;107:1001-10.

8 Laheij RJ, Sturkenboom MC, Hassing RJ, et al. Risk of community-acquired pneumonia and use of gastric acid-suppressive drugs. JAMA 2004;292:1955-60.

9 Sherwood MW, Melloni C, Jones WS, et al. Individual proton pump inhibitors and outcomes in patients with coronary artery disease on dual antiplatelet therapy: a systematic review. J Am Heart Assoc 2015;4:e002245.

10 Kuipers EJ, Lundell L, Klinkenberg-Knol EC, et al. Atrophic gastritis and Helicobacter pylori infection in patients with reflux esophagitis treated with omeprazole or fundoplication. N Engl J Med 1996;334:1018-22.

11 Lundell L, Vieth M, Gibson F, et al. Systematic review: the effects of long-term proton pump inhibitor use on serum gastrin levels and gastric histology. Aliment Pharmacol Ther 2015;42:649-63.

12 Tran-Duy A, Spaetgens B, Hoes AW, et al. Use of proton pump inhibitors and risks of fundic gland polyps and gastric cancer: systematic review and meta-analysis. Clin Gastroenterol Hepatol 2016;14:1706-19.

13 Kuipers EJ, Nelis GF, Klinkenberg-Knol EC, et al. Cure of Helicobacter pylori infection in patients with reflux oesophagitis treated with long term omeprazole reverses gastritis without exacerbation of reflux disease: results of a randomised controlled trial. Gut 2004;53:12-20.

14 Schenk BE, Kuipers EJ, Nelis GF, et al. Effect of Helicobacter pylori eradication on chronic gastritis during omeprazole therapy. Gut 2000;46:615-21.

15 The Hospital Authority. Hospital authority statistical report 2012-2013.. http://www. ha.org.hk/haho/ho/stat/HASR1415_2.pdf (accessed 12 Jan 2017).

16 Chiu SS, Lau YL, Chan KH, et al. Influenza-related hospitalizations among children in Hong Kong. N Engl J Med 2002;347:2097-103.

17 Chan EW, Lau WC, Leung WK, et al. Prevention of dabigatran-related gastrointestinal bleeding with gastroprotective agents: a population-based study. Gastroenterology 2015;149:586-95

18 Cheung KS, Seto WK, Fung J, et al. Epidemiology and natural history of primary biliary cholangitis in the Chinese: a territory-based study in Hong Kong between 2000 and 2015. Clin Trans/ Gastroenterol. In Press. 2017;8:e116.
19 Pratt N, Chan EW, Choi NK, et al. Prescription sequence symmetry analysis: assessing risk, temporality, and consistency for adverse drug reactions across datasets in five countries. Pharmacoepidemiol Drug Saf 2015;24:858-64.

20 Roughead EE, Chan EW, Choi NK, et al. Variation in association between thiazolidinediones and heart failure across ethnic groups: retrospective analysis of large healthcare claims databases in six countries. Drug Saf 2015;38:823-31.

21 Wong OF, Ho PL, Lam SK. Retrospective review of clinical presentations, microbiology, and outcomes of patients with psoas abscess. Hong Kong Med J 2013;19:416-23.

22 Wong AY, Wong IC, Chui CS, et al. Association between acute neuropsychiatric events and helicobacter pylori therapy containing clarithromycin. JAMA Intern Med 2016;176:828-34

$23 \mathrm{Gu}$ Q, Xia HH, Wang JD, et al. Update on clarithromycin resistance in Helicobacter pylori in Hong Kong and its effect on clarithromycin-based triple therapy. Digestion 2006;73:101-6.

24 Hung IF, Chan P, Leung S, et al. Clarithromycin-amoxycillin-containing triple therapy: a valid empirical first-line treatment for Helicobacter pylori eradication in Hong Kong? Helicobacter 2009;14:505-11.

25 Tamim H, Duranceau A, Chen LQ, et al. Association between use of acidsuppressive drugs and risk of gastric cancer. A nested case-control study. Drug Saf 2008;31:675-84.

26 Tamim H, Monfared AA, LeLorier J. Application of lag-time into exposure definitions to control for protopathic bias. Pharmacoepidemiol Drug Saf 2007;16:250-8.

27 Howden CW, Hunt RH. The relationship between suppression of acidity and gastric ulcer healing rates. Aliment Pharmacol Ther 1990;4:25-33.

28 Poulsen AH, Christensen S, McLaughlin JK, et al. Proton pump inhibitors and risk of gastric cancer: a population-based cohort study. Br J Cancer 2009;100:1503-7.

29 García Rodríguez LA, Lagergren J, Lindblad M. Gastric acid suppression and risk of oesophageal and gastric adenocarcinoma: a nested case control study in the UK. Gut 2006;55:1538-44.

30 Thrift AP, Anderson LA, Murray LJ, et al. Nonsteroidal anti-inflammatory drug use is not associated with reduced risk of Barrett's esophagus. Am J Gastroenterol 2016;111:1528-35.

31 Stürmer T, Rothman KJ, Avorn J, et al. Treatment effects in the presence of unmeasured confounding: dealing with observations in the tails of the propensity score distribution - a simulation study. Am J Epidemiol 2010;172:843-54.

32 Svanström H, Pasternak B, Hviid A. Use of azithromycin and death from cardiovascular causes. N Engl J Med 2013;368:1704-12.

33 Kuipers EJ, Uyterlinde AM, Peña AS, et al. Increase of Helicobacter pylori-associated corpus gastritis during acid suppressive therapy: implications for long-term safety. Am J Gastroenterol 1995;90:1401-6.

34 Malfertheiner P, Megraud F, O'Morain CA, et al. Management of Helicobacter pylori infection - the Maastricht IV/ Florence Consensus Report. Gut 2012;61:646-64.

35 Schneider JL, Kolitsopoulos F, Corley DA. Risk of gastric cancer, gastrointestinal cancers and other cancers: a comparison of treatment with pantoprazole and other proton pump inhibitors. Aliment Pharmacol Ther 2016;43:73-82. 